\title{
Fluorescence Imaging System for Biological Tissues Diagnosis: Phantom and Animal Studies
}

\author{
Valery Shupletsov ${ }^{1 *}$, Ksenia Kandurova ${ }^{1}$, Viktor Dremin ${ }^{1,2}$, Elena Potapova ${ }^{1}$, Mikhail Apanaykin ${ }^{3}$, \\ Ulyana Legchenko ${ }^{3}$, and Andrey Dunaev ${ }^{1}$ \\ ${ }^{1}$ Research and Development Center of Biomedical Photonics, Orel State University, 95 Komsomolskaya st., \\ Orel 302026, Russia \\ ${ }^{2}$ Aston Institute of Photonic Technologies, Aston University, Aston Triangle, Birmingham B4 7ET, UK \\ ${ }^{3}$ Department of Industrial Chemistry and Biotechnology, Orel State University, 95 Komsomolskaya st., Orel 302026, \\ Russia \\ * e-mail: valery.shupletsov@bmecenter.ru
}

\begin{abstract}
Currently, optical biopsy is a promising area of diagnosing the state of tissues in real time during the surgical treatment of oncological diseases. The important part of this direction is the development of fluorescence imaging systems and ensuring the accuracy of the calibration of optical measurements. The article describes the development of fluorescence imaging system to define tumor surgical resection margins of abdominal organs. In this study, we proposed of low-cost optical tissue-mimicking phantom combining solid base and liquid part that suitable for quick calibration of fluorescence imaging systems depending on the target endogenous fluorophores. The results of two series of experimental measurements are described. The first measurements of the optical phantom with riboflavin mononucleotide (imitating flavin adenine dinucleotide) and protoporphyrin IX demonstrated the sensitivity of the developed device to proportionally changing concentrations of target fluorophores. The second part of the study included in vivo measurements of liver tumors modeled in mice. The obtained results showed the ability of the developed fluorescence imaging system to register changes in fluorescence due to carcinogenesis. (C) 2020 Journal of Biomedical Photonics \& Engineering.
\end{abstract}

Keywords: optical phantom; optical biopsy; fluorescence imaging; fluorescence spectroscopy; riboflavin; gelatin; porphyrin; hepatocellular carcinoma.

Paper \#3358 received 13 Mar 2020; accepted for publication 19 Mar 2020; published online 24 Mar 2020. doi: 10.18287/JBPE20.06.010303.

\section{Introduction}

According to the World Health Organization statistics, oncological diseases remains the second leading cause of in mortality worldwide [1]. Approximately 18.1 million new cases of cancer were detected in 2018 and 9.6 million deaths from cancer occurred. A significant percentage of incidence and mortality rates is occupied by malignant tumors and their metastases that develop in the abdominal and gastrointestinal organs (liver, pancreas, stomach, esophagus, colon, rectum, etc.). One of the causes leading to deterioration of prognosis in patients is the difficulties in accurate diagnosis of area and depth of malignant infiltration during the surgical treatment.

The leading method for treating many malignant tumors including abdominal and gastrointestinal ones is surgery [2-4]. The surgical treatment is aimed at safely removing as much of the tumor tissue without the necessity of additional surgery as possible. The main challenge surgeons face in this case is accurate definition of resection margins during the surgery. Detection and differentiation of intact and tumor tissue before and during surgical treatment is usually done by palpation and visual examination followed by intraoperative histopathological analysis of samples 
taken from suspicious tissue sites. Visual inspection in white light and palpation are limited in sensitivity and subjectivity of visual and tactile sensations of the surgeon as it is hard to clearly distinguish the presence of the tumor tissue at the places of resection. A procedure express biopsy of each tissue sample takes about 20-25 min, which significantly increases the duration of the surgery and may pose risks to the patient. Moreover, the samples are usually taken randomly from several places of resection edge, which enhances the probability of a false negative result and can lead to cancer reversal in up to $19 \%$ of cases $[5,6]$. In this regard, the problem of developing new diagnostic methods that allow the surgeon to receive additional diagnostic information on localization of malignant tissues in the resection margin in real time remains relevant direction of medical and biomedical engineering research.

Currently, one of the most promising and rapidly developing areas of real-time diagnostics for solving surgical treatment problems is a large number of optical methods often combined in the literature by the term "optical biopsy" [7-10]. These methods are aimed at providing the results comparable to data obtained by traditional methods without the requirement to acquire a tissue sample and spend considerable amount of time to perform the analysis. Optical biopsy includes spectroscopy and imaging techniques used to assess various parameters of morphology and metabolism of biological tissues in vivo [11, 12]. Additional diagnostic information can increase the effectiveness of surgical treatment of cancer in general and provide decision making support for organ preserving treatment.

One of the optical effects widely used to study metabolic activity is fluorescence. Fluorescence diagnostic methods are based on probing the tissue with monochromatic radiation of the near ultraviolet or visible range, exciting the fluorescence of endogenous or exogenous fluorophores and recording secondary emission for further analysis. Two most popular approaches - fluorescence spectroscopy (FS) and fluorescence imaging (FI) - demonstrate significant sensitivity to the presence of pathological changes in tissues, in particular malignant ones [13-15]. Extensive studies have been conducted in this direction for several decades to develop methodologies and devices for effective distinguishing of normal and tumor tissues in various human organs (lungs, breast, colon, cervix, etc.) [16-18].

Currently, a number of systems for fluorescenceguided surgery have already been clinically tested and implemented in the practice of surgeons. However, many of these systems involve the use of special fluorescent dyes for optical diagnostics in the near infrared range [19]. On the other hand, changes in autofluorescence caused by altered metabolic processes are also studied for the purpose of malignancies diagnosis $[10,20,21]$.

It is known that cells undergo malignant transformations during carcinogenesis proceed which lead to morphological and biochemical changes detectable by fluorescence methods. The metabolic rate activity affects the content of certain fluorophores, such as nicotinamide adenine dinucleotide (NAD) and flavin adenine dinucleotide (FAD). These coenzymes are contained in the cytosol and mitochondria and are involved in reactions providing cells with energy, acting as electron donors and acceptors. These substances undergo oxidation and reduction reactions and reduced NAD (NADH) and oxidized FAD demonstrate the greatest contribution to tissue fluorescence. Maximum NADH fluorescence emits in blue light under $365 \mathrm{~nm}$ excitation, while FAD fluorescence emits in green light under $450 \mathrm{~nm}$ excitation [20]. Several clinical studies show that oncological processes affect such parameters as fluorescence intensity and redox ratio (FAD/NADH) [22]. Active proliferation in hypoxia forces tumor cells to switch to a glycolytic type of metabolism. These conditions lead to an increase in the concentration of $\mathrm{NADH}$, change in redox ratio of FAD and NADH (FAD/NADH) [23-25]. Several clinical studies of FAD in tumors showed the changes in its fluorescence associated with oncological processes as well [26-28]. As FAD is involved in several metabolic reactions (tricarboxylic acid cycle, oxidative phosphorylation, acetyl coenzyme A synthesis, etc.) [29], the increase in FAD fluorescence can be explained by an increase in metabolic activity in abnormal tissues, which indicates tumor growth [30].

It is also reported that oncological processes cause abnormal increase of porphyrins accumulation, which indicates specific metabolic disorders of tumor cells [23, 31]. Porphyrin, namely protoporphyrin IX (PpIX), accumulates in tumors more actively [32-34], which is widely used in photodynamic therapy. Autofluorescence diagnostics of porphyrins has sufficient sensitivity to the presence of tumor sites as well [35].

Thus, the contribution of the listed fluorophores (NADH, FAD, porphyrins) to the spectra of endogenous fluorescence is closely dependent on the state of the tissue, which indicates the relevance of the development of methodologies and technical implementation for their registration and analysis.

While the FS and FI methods can be successfully used for the fluorescence contrasting at the selected wavelength of the emission [36], the fluorescence measurements of endogenous fluorophores in living tissues can be challenging due to the overlap of the emission spectra of different fluorophores. Moreover, biological tissues are heterogeneous complex structures containing various fluorophores in different concentrations and at different depths [37, 38]. In order to correctly interpret the experimental data in future clinical applications, it is necessary to determine the contribution of each component to the total signal. For this purpose, researchers usually perform test measurements using normal or pathologically altered tissues of laboratory animals or specially designed optical phantoms. While the studies in model animals are more close to real conditions of clinical in vivo 
measurements, they are also more resource consuming and it is harder to imitate certain optical properties. Optical phantoms allow one to mimic the typical interaction of optical radiation with biological tissue with determined parameters of absorption, scattering, fluorescence, etc., which can be implemented both as test method at the stage of development and as the calibration method for future routine running in practice. The images of optical phantoms can be also used to improve resolution and quantification of uncorrected images using, for example, the deconvolution method [39].

Currently, researchers use several forms of optical phantoms in their studies in order to reach the versatility of properties mimicked, decrease the cost of the phantom and ensure the quality conform to the application goal. Most of the phantom spectroscopic studies available in literature are based on the development of liquid phantoms, which are used for modelling infinite or semi-infinite single layer media with absorption, scattering and fluorescence effects [40, 41]. For imitation of soft tissue-mimicking fluorescence gel-wax phantom based on mineral-oil material is known to be created [42].

The concept of a thin solid optical tissue phantom to maintain the required geometrical shape, thickness and inhomogeneity similar to multi-layered tissues is a challenging task. Various materials are used to create this phantoms, such as epoxy, polyester resin, or polyurethane material, which provide the photostability needed in long-term imaging applications [43-45]. Several scientific studies describe single-layer and twolayer solid gelatin phantoms fabricated for fluorescence studies, which are used to mimic normal and dysplasia tissue conditions [46, 47]. In this case, gelatin is used to imitate the fluorescence of collagen, as this protein is the main structural component of connective tissues.

Therefore, the aim of this work was the development of FI system and an optical tissue-mimicking phantom for its calibration as a preliminary stage of complex development of FI technology for detecting tumor margins during the surgery. As a target endogenous fluorophores for this study, FAD and PpIX were chosen. As simplicity and accessibility should play an important role in development and using of this technology, the main objective of this study was to assess the possibilities of development of low-cost phantom.

\section{Materials and Methods}

\subsection{Experimental Setup}

The scheme of the experimental home-built setup developed in this study is presented in Fig. 1.

The excitation channel included $455 \mathrm{~nm}$ LLS-455 LED source (Ocean Optics, USA) to excite the endogenous fluorescence. The radiation from the source was directed through a condenser and MF445-45 bandpass filter (Thorlabs, Inc. USA) to the object to cut a narrow band of excitation and minimize the effect of other tissue fluorophores. The diameter of the illuminated spot was adjusted with a collimator in the range from 1 to $3 \mathrm{~cm}$. The image recording channel included FGL495 and FGL610 glass filters (Thorlabs, Inc. USA) with cut-off wavelengths of $495 \mathrm{~nm}$ and 610 $\mathrm{nm}$ to attenuate backscattered source radiation and pass the certain bands of fluorescence radiation to the detector. The images were recorded by $340 \mathrm{M}-\mathrm{USB}$ fast frame rate monochrome scientific $\mathrm{CCD}$ camera (Thorlabs, Inc. USA). The camera's area of interest was $2.5 \mathrm{~cm}$ in diameter. The camera is controlled by the standard ThorCam software (Thorlabs, Inc. USA).

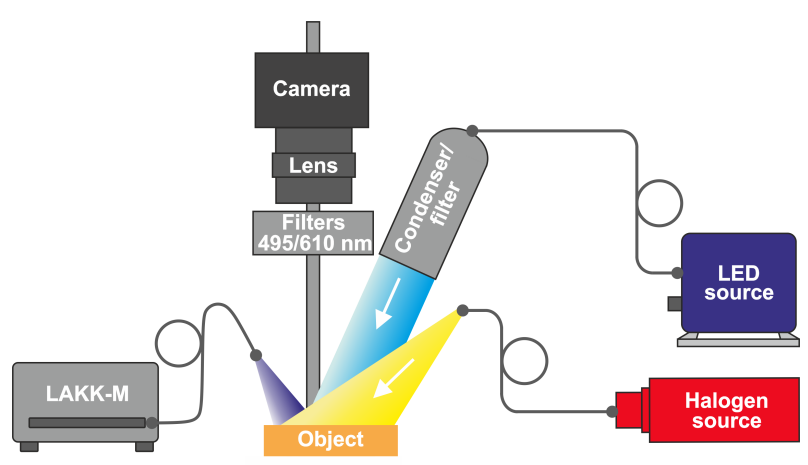

Fig. 1 The scheme of the experimental setup.

The FS method used for additional control measurements was implemented by the multifunctional laser diagnostic complex LAKK-M (SPE "LAZMA" Ltd, Russia). This device provided fluorescence excitation at $365 \mathrm{~nm}$ and $450 \mathrm{~nm}$ wavelengths and was equipped with $3 \mathrm{~mm}$ optical fiber probe $(400 \mu \mathrm{m}$ fibers, $\mathrm{NA}=0.22$ ). The spectra were recorded by the built-in spectrometer in the range of 340-800 nm.

In addition, the stabilized broadband radiation source of 360-2600 $\mathrm{nm}$ range SLS201L-M (Thorlabs Inc., USA) was used to record white light images and compare them with fluorescence images.

\subsection{Tissue-mimicking Phantom Development}

The idea of the optical phantom designed in this work was to combine the solid base for modeling of background collagen fluorescence and the liquid part to mimic the various concentrations of FAD and porphyrin fluorescence in biological tissues.

To produce optical phantoms imitating collagen fluorescence, it is possible to use gelatin, which is its hydrolyzed form. In this work, we created a multi-layer model consisting of sheets of gelatin with a thickness of less than $1 \mathrm{~mm}$ and a weight of less than $5 \mathrm{~g}$. The optimal number of gelatin sheets was experimentally selected by comparing the fluorescence intensity levels of gelatin sheets and skin collagen. To simulate the FAD fluorescence spectrum, a $1 \%$ solution $(26.6 \mu \mathrm{M} / \mathrm{g})$ of riboflavin mononucleotide was used. This substance belongs to the flavin group and has the fluorescence spectrum similar to the spectrum of FAD. Riboflavin has a higher quantum yield, and, unlike FAD, its quantum yield within $\mathrm{pH} 4-8$ does not depend on acidity [48]. It is known that the concentration of FAD 
in human body usually varies from 0.1 to $1 \mu \mathrm{M} / \mathrm{g}$ [49]. In accordance with this data, four concentrations of riboflavin were selected close to the real concentrations of FAD in biological tissues. The solution of riboflavin mononucleotide was diluted with distilled water in the following ratios: 1:32, 1:64, 1:128 and 1:256. Thus, the solid base of the optical phantom was composed of two sheets of gelatin. The liquid part was made of the solutions of four different concentrations $(0.84 ; 0.42$; $0.21 ; 0.1 \mu \mathrm{M} / \mathrm{g}$ ) of riboflavin distributed in four different areas of the phantom.

In our research, we used PpIX obtained chemically from the dark eggshell. It is known that PpIX, an immediate precursor of heme, is the main pigment that create the shell color [50]. Before the extraction, the eggshells were thoroughly washed of albumen and shell membranes and dried. $10 \mathrm{~g}$ of the eggshells were mixed with $150 \mathrm{ml}$ of a $1 \mathrm{M}$ hydrochloric acid solution. The reacting mixture was maintained until the release of carbon dioxide ceased. The undissolved residue was separated by filtration through a paper filter. The resulting emulsion was centrifuged to completely separate the layers. The top layer containing PpIX was transferred to a conical flask and $10 \mathrm{~g}$ of pre-calcined sodium sulfate was added to PpIX. The PpIX solution was separated from the salt by decantation and evaporated in a hot water bath until the solvent was completely removed. The residue was dissolved in $5 \mathrm{ml}$ of dimethyl sulfoxide. As a result, PpIX was obtained at a concentration of $0.3 \mu \mathrm{M} / \mathrm{g}$, which was applied to two sheets gelatin model similar to riboflavin.

The research methodology included sequential registration of fluorescence images using the CCD camera and the spectrometer. Drops of the same volume $(20 \mu \mathrm{l})$ with different concentrations of riboflavin or PpIX were applied to gelatin sheets using a micropipette. The resulting phantoms with riboflavin and PpIX were located on a non-fluorescent nonreflective surface. The registration of images was carried out in the following order. At the first stage, a $495 \mathrm{~nm}$ filter was installed in front of the camera, and fluorescence images were recorded 3 times. Then the image of phantoms in white light was recorded without

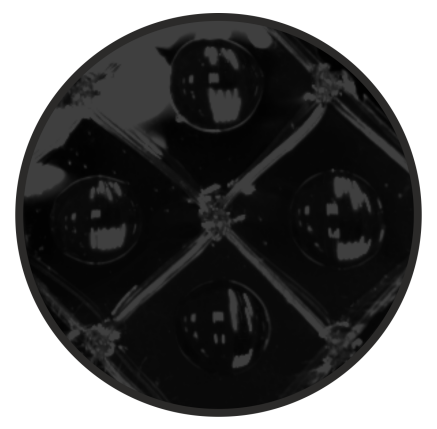

Fig. 2 Obtained images of the optical phantom: original image of riboflavin phantom in white light (a), fluorescence
image of riboflavin phantom (b), fluorescence image of PpIX phantom (c).

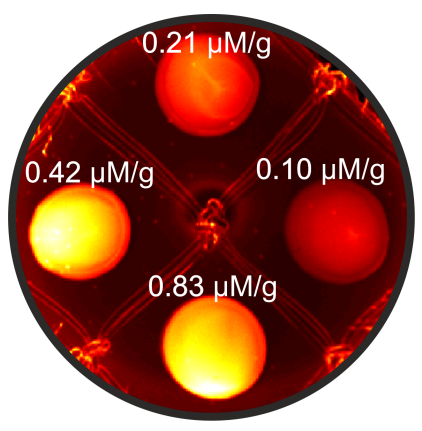

Fig. 2 Obtained images of the optical phantom: original image of riboflavin phantom in white light (a), fluorescence
image of riboflavin phantom (b), fluorescence image of PpIX phantom (c).

a filter. At the second stage, the fluorescence spectra of each phantom were recorded 3 times. The results were processed on a personal computer using specially developed software in the Matlab environment.

\subsection{Animals Model}

The developed FI system demonstrated a rather high sensitivity to changes in the concentrations of dysplasia markers, such as flavins and porphyrins. In connection with this next step was also testing an animal model of liver cancer. The studies were approved by the Ethics Committee of Orel State University named after I.S. Turgenev (protocol No. 10 of 16.10.2017) and were carried out in accordance with the principles of Good Laboratory Practice [51]. Optical measurements were conducted in the BDF (C57B16xDBA) laboratory mice (male, $\mathrm{n}=2,20$ weeks). H33 mouse hepatocellular carcinoma culture was previously implanted through a syringe into the medial lobe of the liver in a volume of $100 \mu 1(50,000$ cells $/ \mu 1)$ per mouse during abdominal surgery. After approximately 2 months after the surgery, the experiments were carried out as mice increased their weight by more than $15 \%$ of the normal weight.

Before the measurements, the animal was anesthetized with Zoletil (Vibrac, France) in the standard dosage. The animal was fixed on a special platform in the back position. A transverse laparotomy was performed to gain access to the abdominal cavity. A surgeon performed a visual and palpatory examination of the abdominal cavity to determine the presence and localization of malignant and healthy tissue in the liver and other organs. The next step was to place the animal under the imaging channel of the experimental set up to adjust the focus, frame rate and exposure time according to the area of interest.

The research methodology included step-by-step registration of liver and tumor images in white light without filters and under $450 \mathrm{~nm}$ excitation with optical filters to obtain fluorescence images. The measurements were repeated 3 times. The obtained data was processed on a personal computer using custom developed software in the Matlab computing environment. image of riboflavin phantom (b), fluorescence image of PpIX phantom (c).

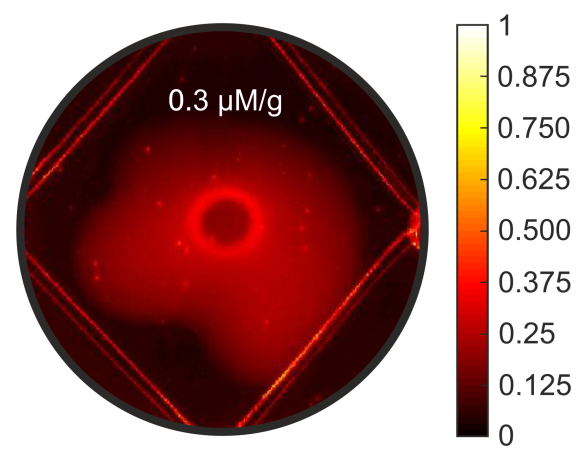

(c) 


\section{Results and Discussion}

\subsection{Tissue-mimicking Phantom}

As a result, the registered images of created optical phantom under halogen lamp illumination and LED illumination after preliminary processing are presented in Fig. 2a-c. The white light images (Fig. 2a) were recorded with exposure time of $20 \mathrm{~ms}$, fluorescence images (Fig. 2b, c) were recorded with $400 \mathrm{~ms}$ exposure time. After that, the intensities were normalized to the maximum intensity to represent the obtained data in the range of $[0 ; 1]$. The fluorescence images of PpIX liquid on the gelatin surface of developed phantom was also recorded (Fig. 2c).

The intensity values of riboflavin and PpIX fluorescence were averaged in similar areas of each drop. The maximum fluorescence intensities obtained with the FS method were averaged and compared with the results of FI. Thereafter, the intensities were normalized by the maximum obtained value, so the maximum intensity was equal to 1 . The results of fluorescence intensities measurements for each concentration of riboflavin and PpIX using FS modality are presented in Fig. 3.

For the three lowest concentrations of riboflavin, it was noticed that the fluorescence intensity recorded by FI decreases proportionally to the decrease of concentrations. However, the same trend was not observed for the highest concentration, which can be caused by reaching such concentration, starting from which the level of fluorescence intensity becomes constant.

The trend calculated from fluorescence images (Fig. 3c) was also confirmed during measurements with FS channel. Future studies will be dedicated to the combining of the fluorescence measurements with hyperspectral technology [52] to develop effective compensation algorithms to attenuate the influence of tissue/phantom light absorbers (e.g. hemoglobin) on the fluorescence intensity recorded.

Based on the results presented in Fig. 2 and 3c, a significant effect of uneven illumination of the optical phantom was observed during data processing, which suggests the implementation of orthogonal optical schemes in future research.

\subsection{Animal Study}

In the course of the study, images of the abdominal cavity of two laboratory animals with an experimental malignant tumor of the liver were obtained.

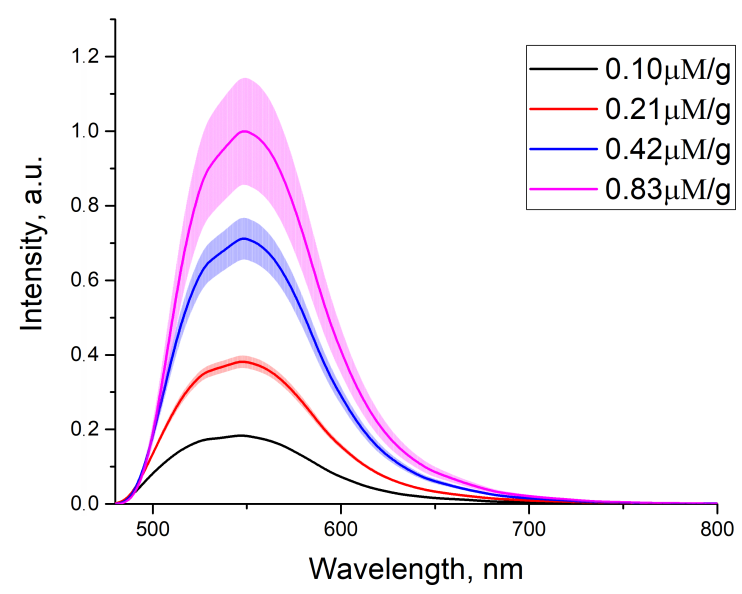

(a)

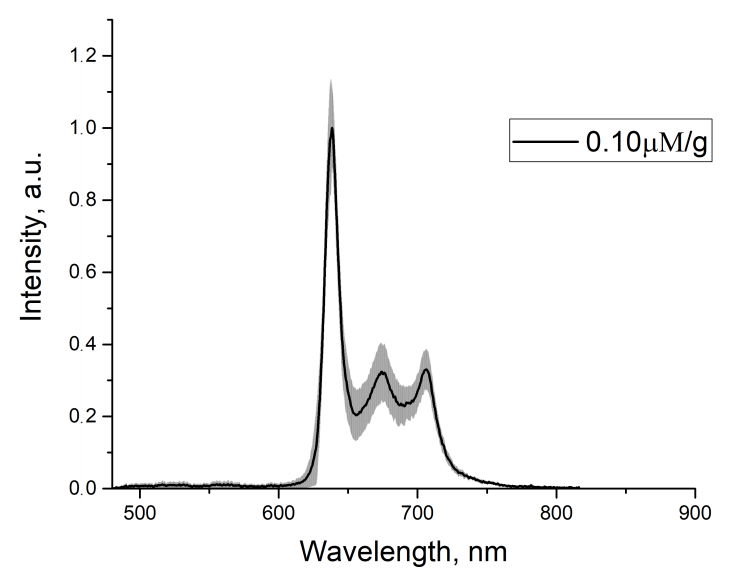

(b)

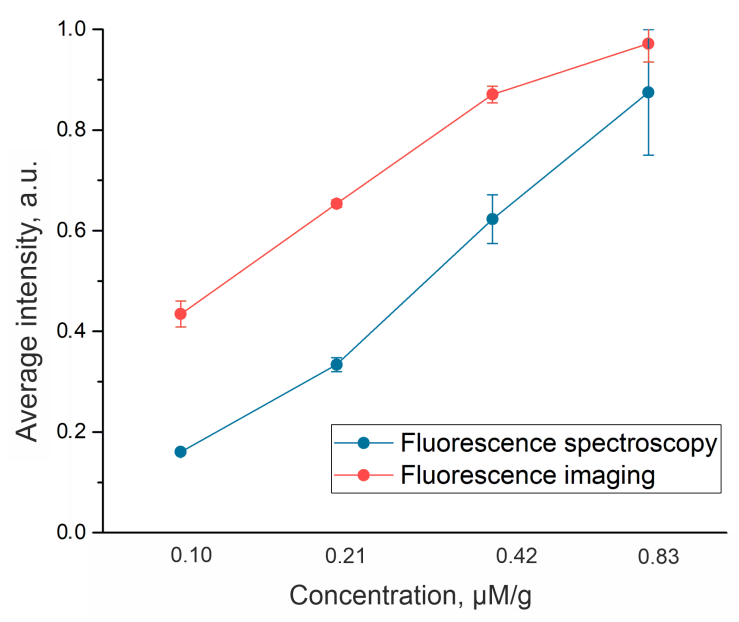

(c)

Fig. 3 Average normalized fluorescence spectra of riboflavin (a) and PpIX (b) solutions, fluorescence intensities curves obtained from FS and FI data (c). 


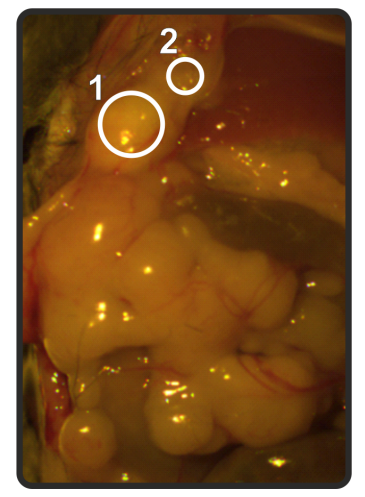

(a)

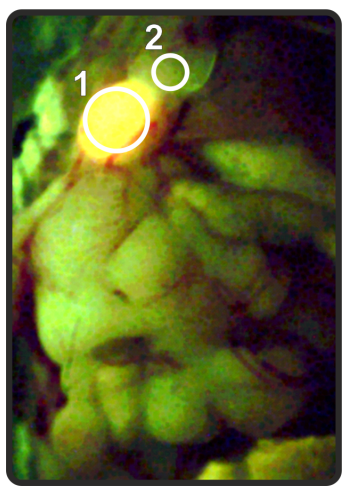

(b)

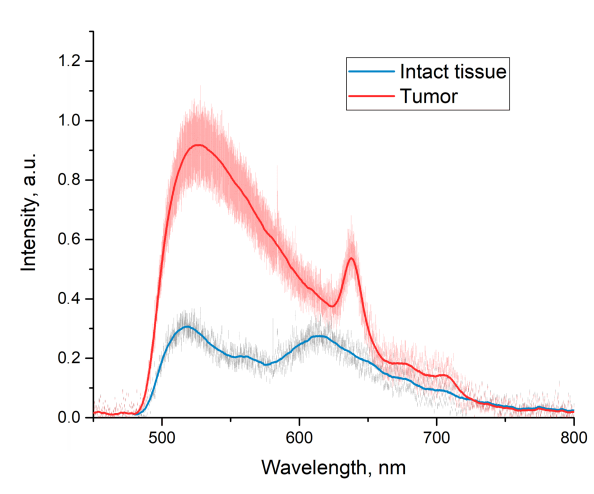

(c)

Fig. 4 The image in white light (a), fluorescence image with $495 \mathrm{~nm}$ filter (b) and fluorescence spectra (c) of abdominal cavity of mouse 1 .

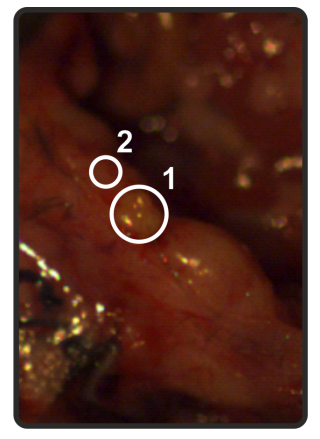

(a)

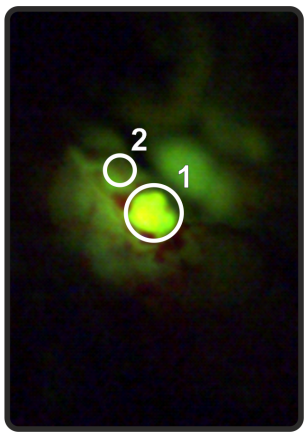

(b)

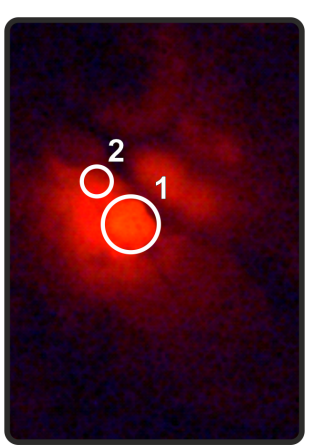

(c)

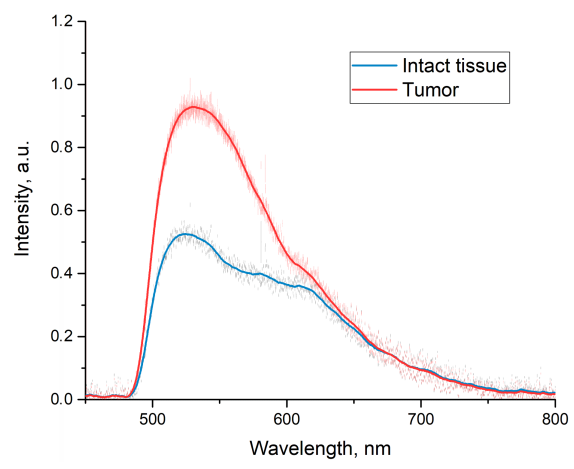

(d)

Fig. 5 The image in white light (a), fluorescence image with $495 \mathrm{~nm}$ filter (b), fluorescence image with $610 \mathrm{~nm}$ filter (c) and fluorescence spectra (d) of abdominal cavity of mouse 2.

Fig. 4 and 5 show white images, fluorescence images and fluorescence spectra of target zones. The areas highlighted by a circle 1 correspond to the localization of the tumor. The areas highlighted by a circle 2 correspond to surrounding intact liver tissues. When the light is filtered with $495 \mathrm{~nm}$ filter, the fluorescence can be visualized mainly in the range of $500-540 \mathrm{~nm}$. With $610 \mathrm{~nm}$ filter, a clear contrast of fluorescence is observed in the range of 620-640 nm.

It should be noted that fluorescence images of mouse 1 were obtained only with $495 \mathrm{~nm}$ light filter. The diameter of the probing area was about $3 \mathrm{~cm}$. During the analysis of the initial images of mouse 1, it was noted that the tumor was not green colored as it should be for the peak of FAD fluorescence (about $530 \mathrm{~nm}$ ). Instead, it was yellow, which was presumably associated with significant accumulation of porphyrins in the tumor and, thus, the summation of fluorescence FAD and porphyrins (fluorescence in the range of 620 $640 \mathrm{~nm}$ ). The FS data confirmed this hypothesis; therefore, FI measurements protocol and experimental setup were upgraded for the second series of measurements. The images of mouse 2 were obtained using two filters.

The values of white light reflectance intensity and fluorescence intensity in the highlighted areas were averaged to compare the data from the tumor and surrounding tissues obtained in white light and fluorescence mode. As a result, it was found that while the intensity of the tumor reflectance exceeded the intensity from unchanged tissue by 1.2 times. Fluorescence images demonstrated the value of this parameter is 4.2 for $495 \mathrm{~nm}$ filtered images and 2.6 for $610 \mathrm{~nm}$ filtered images. This result proves that it is possible to more clearly distinguish the tumor focus. An increase of fluorescence intensity in malignant tissues compared with the surrounding tissues in $495 \mathrm{~nm}$ filtered images may indicate changes in the content of endogenous fluorophores, which in turn are caused by metabolic disorders accompanying carcinogenesis process.

The images of mouse 2 under $495 \mathrm{~nm}$ filter did not demonstrate the same pronounced yellow color in the tumor. The fluorescence spectra (Fig. 4c and Fig. 5d) demonstrated that the accumulation of PpIX fluorescence in the tumor of mouse 1 was greater than in mouse 2. Based on the analysis of the obtained images and spectra, it was noticed that the carcinogenesis processes occurred individually in each animal, which should also be taken into account when planning and conducting further experimental studies. 
Thus, the obtained images and the calculated differences in the tumor/intact tissue ratio of average fluorescence intensity indicate a high sensitivity of FI technique to the presence of malignant changes in the tissues compared to conventional images recorded in white light. Depending on the fluorescence intensity increase degree, a more accurate determination of the accumulation sites of various fluorophores in the tissue structure of the organ is possible in vivo. At the same time, the FS method can also provide additional information for the correct interpretation of the FI data. In this regard, the use of hyperspectral technologies for measuring the fluorescence of biological tissues is promising.

\section{Conclusion}

Many researchers work on the development of technical implementation and diagnostic criteria for intraoperative fluorescence imaging based on the autofluorescence of endogenous fluorophores. An important stage of such research is the providing of reproducible measurements of biological tissues for both the process of development itself and for primary and periodic calibration of imaging systems during their practical application.

For these purposes, we developed the experimental setup for fluorescence imaging of tissues fluorophores associated with metabolic changes and carcinogenesis. The optical phantom mimicking the biological tissue fluorescence was also created for its calibration as a preliminary stage of complex development of FI technology for cancer detection during surgery.

These studies showed the possibility of application of the developed fluorescence imaging system for obtaining real-time information about the presence of malignant cells on the resection margin in cancer surgery. The implementation of FI technology to clinical practice can increase the effectiveness of surgical interventions in the long term by reducing time spend on surgeries and reducing the probability of a false negative error during the defining of the resection margin.
The proposed phantom is relatively simple to manufacture and use for measurements. The phantom mimics tissue fluorescence for specific research tasks, taking into account the presence of collagen. As an option for further research in this direction, it is planned to develop a more complex optical model of biological tissue using other endogenous fluorophores, such as NADH and FAD themselves. The obtained experimental data indicate the sensitivity of the developed fluorescence phantom to changes in the concentration of flavins, as well as the presence of PpIX.

Application of the developed optical phantom will allow to more accurately test the FI systems and improve the overall quality of implementation of this technology. The data obtained will be used to correct fluorescence images of pathological biological tissues and to more clearly determine the resection margins. Further research will be aimed at improving the technical implementation and the optical phantom, as well as conducting reliable and reproducible experimental measurements of normal and tumor tissue parameters to develop diagnostic criteria and introduce fluorescence imaging technology into clinical practice.

\section{Disclosures}

All authors declare that there is no conflict of interests in this paper.

\section{Acknowledgments}

The study was supported by the Russian Foundation for Basic Research according to the research project 18-0200669. VD acknowledges for personal support from the European Union's Horizon 2020 research and innovation program under the Marie Sklodowska-Curie grant agreement No. 839888. Authors are very grateful to Dr. Olga V. Morozova (N. N. Blokhin National Medical Research Center of Oncology, Moscow, Russia) for tumor cell inoculation.

\section{References}

1. R. F. Bray, J. Ferlay, I. Soerjomataram, R. L. Siegel, L. A. Torre, and A. Jemal, "Global cancer statistics 2018: GLOBOCAN estimates of incidence and mortality worldwide for 36 cancers in 185 countries," CA: A Cancer Journal for Clinicians 68(6), 394-424 (2018).

2. S. Lin, K. Hoffmann, and P. Schemmer, "Treatment of hepatocellular carcinoma: a systematic review," Liver cancer 1(3-4), 144-158 (2012).

3. N. Coburn, R. Cosby, L. Klein, G. Knight, R. Malthaner, J. Mamazza, C. D. Mercer, and J. Ringash, "Staging and surgical approaches in gastric cancer: A systematic review," Cancer Treatment Reviews 63, 104-115 (2018).

4. K. D. Miller, L. Nogueira, A. B. Mariotto, J. H. Rowland, K. R. Yabroff, C. M. Alfano, A. Jemal, J. L. Kramer, and R. L. Siegel, "Cancer treatment and survivorship statistics, 2019," CA: A Cancer Journal for Clinicians 69(5), 363385 (2019).

5. K. J. Chambers, S. Kraft, and K. Emerick, "Evaluation of frozen section margins in high-risk cutaneous squamous cell carcinomas of the head and neck," Laryngoscope 125(3), 636-639 (2015).

6. E. L. Rosenthal, J. M. Warram, E. de Boer, J. P. Basilion, M. A. Biel, M. Bogyo, M. Bouvet, B. E. Brigman, Y. L. Colson, S. R. DeMeester, G. C. Gurtner, T. Ishizawa, P. M. Jacobs, S. Keereweer, J. C. Liao, Q. T. Nguyen, J. M. 
Olson, K. D. Paulsen, D. Rieves, B. D. Sumer, M. F. Tweedle, A. L. Vahrmeijer, J. P. Weichert, B. C. Wilson, M. R. Zenn, K. R. Zinn, and G. M. van Dam, "Successful translation of fluorescence navigation during oncologic surgery: a consensus report," Journal of Nuclear Medicine 57(1), 144-150 (2016).

7. I. J. Bigio, J. R. Mourant, “Optical biopsy," in Encyclopedia of Optical Engineering, CRC Press, Boca Raton, 1577-1593 (2003).

8. T. D. Wang, J. Van Dam, “Optical biopsy: A new frontier in endoscopic detection and diagnosis," Clinical Gastroenterology and Hepatology 2(9), 744-753 (2004).

9. G. T. Kennedy, O. T. Okusanya, J. J. Keating, D. F. Heitjan, C. Deshpande, L. A. Litzky, S. M. Albelda, J. A. Drebin, S. Nie, P. S. Low, and S. Singhal, "The optical biopsy: A novel technique for rapid intraoperative diagnosis of primary pulmonary adenocarcinomas," Annals of Surgery 262(4), 602-609 (2015).

10. A. C. Croce, A. Ferrigno, G. Bottiroli, and M. Vairetti, "Autofluorescence-based optical biopsy: An effective diagnostic tool in hepatology," Liver International 38(7), 1160-1174 (2018).

11. I. Georgakoudi, M. S. Feld, "The combined use of fluorescence, reflectance, and light-scattering spectroscopy for evaluating dysplasia in Barrett's esophagus," Gastrointestinal Endoscopy Clinics of North America 14(3), 519-537 (2004).

12. S. Y. Lee, W. R. Lloyd, M. Chandra, R. H. Wilson, B. McKenna, D. Simeone, J. Scheiman, and M.-A. Mycek, "Characterizing human pancreatic cancer precursor using quantitative tissue optical spectroscopy," Biomedical Optics Express 4(12), 2828-2834 (2013).

13. A. Gisbrecht, E. Borisova, T. Genova-Hristova, P. Troyanpva, E. Pavlova, N. Penkov, I. Bratchenko, V. Zakharov, I. Lihachova, I. Kuzmina, and J. Spigulis, "Multispectral autoflourescence detection of skin neoplasia using steadystate techniques," Proceeding of SPIE 11047, 1104704 (2019).

14. Y. A. Khristoforova, I. A. Bratchenko, O. O. Myakinin, D. N. Artemyev, A. A. Moryatov, A. E. Orlov, S. V. Kozlov, and V. P. Zakharov, "Portable spectroscopic system for in vivo skin neoplasms diagnostics by Raman and autofluorescence analysis,” Journal of Biophotonics 12(4), e201800400 (2019).

15. K. Kandurova, V. Dremin, E. Zherebtsov, E. Potapova, A. Alyanov, A. Mamoshin, Y. Ivanov, A. Borsukov, and A. Dunaev, "Fiber-optic system for intraoperative study of abdominal organs during minimally invasive surgical interventions," Applied Sciences 9(2), 217 (2019).

16. V. R. Kolli, A. R. Shaha, H. E. Savage, P. G. Sacks, M. A. Casale, and S. P. Schantz, "Native cellular fluorescence can identify changes in epithelial thickness in-vivo in the upper aerodigestive tract," The American Journal of Surgery 170(5), 495-498 (1995).

17. B. Hill, S. F. Lam, P. Lane, C. MacAulay, L. Fradkin, and M. Follen, "Established and emerging optical technologies for the real-time detection of cervical neoplasia: a review," Journal of Cancer Therapy 8(13), 12411278 (2017).

18. K. He, L. Zhao, Y. Chen, X. Huang, Y. Ding, H. Hua, L. Liu, X. Wang, M. Wang, Y. Zhang, and Z. Fan, “Label free multiphoton microscopic imaging as a novel real - time approach for discriminating colorectal lesions: A preliminary study," Journal of Gastroenterology and Hepatology 34(12), 2144-2151 (2019).

19. T. Nagaya, Y. A. Nakamura, P. L. Choyke, and H. Kobayashi, "Fluorescence-guided surgery," Frontiers in Oncology 7, 314 (2017).

20. K. Koenig, H. Schneckenburger, "Laser-induced autofluorescence for medical diagnosis," Journal of Fluorescence 4(1), 17-40 (1994).

21. S. Palmer, K. Litvinova, A. Dunaev, J. Yubo, D. McGloin, and G. Nabi, "Optical redox ratio and endogenous porphyrins in the detection of urinary bladder cancer: A patient biopsy analysis," Journal of Biophotonics 10(8), 1062-1073 (2017).

22. T. Vo-Dinh, Biomedical photonics handbook: biomedical diagnostics, CRC press, Boca Raton (2014).

23. K. T. Moesta, B. Ebert, T. Handke, D. Nolte, C. Nowak, W. E. Haensch, R. K. Pandey, T. J. Dougherty, H. Rinneberg, and P. M. Schlag, "Protoporphyrin IX occurs naturally in colorectal cancers and their metastases," Cancer Research 61(3), 991-999 (2001).

24. C. J. Fox, P. S. Hammerman, and C. B. Thompson, "Fuel feeds function: energy metabolism and the T-cell response," Nature Reviews Immunology 5(11), 844-852 (2005).

25. E. Currie, A. Schulze, R. Zechner, T. C. Walther, and R. V. Farese, "Cellular fatty acid metabolism and cancer," Cell Metabolism 18(2), 153-161 (2013).

26. Y. Wu , P. Xi, J. Y. Qu, T.-H. Cheung, and M.-Y. Yu, "Depth-resolved fluorescence spectroscopy of normal and dysplastic cervical tissue," Optics Express 13(2), 382-388 (2005).

27. Y. Wu, J. Y. Qu, “Autofluorescence spectroscopy of epithelial tissues," Journal of Biomedical Optics 11(5), 054023 (2006).

28. N. Ramanujam, "Fluorescence spectroscopy of neoplastic and non-neoplastic tissues," Neoplasia 2(1-2), 89-117 (2000).

29. M. M. Lukina, M. V. Shirmanova, T. F. Sergeeva, and E. V. Zagaynova, "Metabolic imaging in the study of oncological processes," Sovremennye Tehnologii v Medicine 8(4 (eng)), 113-124 (2016). 
30. I. Pavlova, K. Sokolov, R. Drezek, A. Malpica, M. Follen, and R. Richards-Kortum, "Microanatomical and Biochemical Origins of Normal and Precancerous Cervical Autofluorescence Using Laser - scanning Fluorescence Confocal Microscopy," Photochemistry and Photobiology 77(5), 550-555 (2003).

31. A. C. Croce, G. Santamaria, U. D. Simone, F. Lucchini, I. Freitas, and G. Bottiroli, "Naturally-occurring porphyrins in a spontaneous-tumour bearing mouse model," Photochemical \& Photobiological Sciences 10(7), 1189-1195 (2011)..

32. W. Kemmner, K. Wan, S. Rüttinger, B. Ebert, R. Macdonald, U. Klamm, and K. T. Moesta, "Silencing of human ferrochelatase causes abundant protoporphyrin-IX accumulation in colon cancer," The FASEB Journal 22(2), 500509 (2008).

33. M. Sachar, K. E. Anderson, and X. Ma, "Protoporphyrin IX: the good, the bad, and the ugly," Journal of Pharmacology and Experimental Therapeutics 356(2), 267-275 (2016).

34. E. A. Machinskaya, V. I. Ivanova-Radkevich, "Review of selective accumudation of photosensitizers with different chemical structure in tumor tissue," Photodynamic therapy and photodyagnosis 2(4), 28-32 (2013). [in Russian]

35. A. Moiyadi, P, Shetty, E. Sridhar, V. Gota, M. Gurjar, G. Saicharan, V. Singh, and S. Srivastava, "Objective assessment of intraoperative tumor fluorescence reveals biological heterogeneity within glioblastomas: a biometric study," Journal of Neuro-Oncology 146(3), 477-488 (2020).

36. O. Stelmashchuk, E. Zherebtsov, A. Zherebtsova, E. Kuznetsova, A. Vinokurov, A. Dunaev, A. Mamoshin, I. Snimshchikova, A. Borsukov, A. Bykov, and I. Meglinski, "Noninvasive control of the transport function of fluorescent coloured liposomal nanoparticles," Laser Physics Letters 14(6), 065603 (2017).

37. I. V. Meglinski, S. J. Matcher, "Quantitative assessment of skin layers absorption and skin reflectance spectra simulation in the visible and near-infrared spectral regions," Physiological Measurement 23(4), 741 (2002).

38. P. Thueler, I. Charvet, F. Bevilacqua, M. St. Ghislain, G. Ory, P. Marquet, P. Meda, B. Vermeulen, and C. Depeursinge, "In vivo endoscopic tissue diagnostics based on spectroscopic absorption, scattering, and phase function properties," Journal of Biomedical Optics 8(3), 495-504 (2003).

39. M. Anastasopoulou, D. Gorpas, M. Koch, E. Liapis, S. Glasl, U. Klemm, A. Karlas, T. Lasser, and V. Ntziachristos, "Fluorescence imaging reversion using spatially variant deconvolution," Scientific Reports 9(1), 111 (2019).

40. L. H. Luthjens, T. Yao, and J. M. Warman, “A polymer-gel eye-phantom for 3D fluorescent imaging of millimetre radiation beams," Polymers 10(11), 1195 (2018).

41. H. Lu, F. Floris, M. Rensing, and S. Andersson-Engels, "Fluorescence spectroscopy study of protoporphyrin IX in tissue-like phantoms," Proceeding of SPIE 11190, 111901S (2019).

42. Y. Xie, E. Maneas, S. Islam, W. Peveler, J. Shapey, W. Xia, S. Ourselin, I. P. Parkin, A. E. Desjardins, and T. Vercauteren, "Soft optically tuneable fluorescence phantoms based on gel wax and quantum dots: a tissue surrogate for fluorescence imaging validation," Proceeding of SPIE 10862, 108621F (2019).

43. U. Resch-Genger, M. Grabolle, S. Cavaliere-Jaricot, R. Nitschke, and T. Nann, "Quantum dots versus organic dyes as fluorescent labels," Nature Methods 5(9), 763-775 (2008).

44. M. Anastasopoulou, M. Koch, V. Ntziachristos, D. Gorpas, P. B. Garcia-Allende, U. Klemm, and A. Karlas, "Multiparameter solid phantom for fluorescence imaging standardization," Proceeding of SPIE 10411, 104110J (2017).

45. B. W. Pogue, M. S. Patterson, "Review of tissue simulating phantoms for optical spectroscopy, imaging and dosimetry," Journal of Biomedical Optics 11(4), 041102 (2006).

46. B. Leh, R. Siebert, H. Hamzeh, L. Menard, M.-A. Duval, Y. Charon, and D. A. Haidar, "Optical phantoms with variable properties and geometries for diffuse and fluorescence optical spectroscopy," Journal of Biomedical Optics 17(10), 108001 (2012).

47. K. B. Nivetha, N. Sujatha, "Development of thin skin mimicking bilayer solid tissue phantoms for optical spectroscopic studies,” Biomedical Optics Express 8(7), 3198-3212 (2017).

48. P. Drössler, W. Holzer, A. Penzkofer, and P. Hegemann, " $\mathrm{pH}$ dependence of the absorption and emission behaviour of riboflavin in aqueous solution," Chemical Physics 282(3), 429-439 (2002).

49. A. Aliverti, B. Curti, and M. A. Vanoni, "Identifying and quantitating FAD and FMN in simple and in iron-sulfurcontaining flavoproteins," in Flavoprotein Protocols, S. K. Chapman, G. A. Reid (eds.), Springer, New York, 9-23 (1999).

50. M. L. Dean, T. A. Miller, and C. Brückner, "Egg-citing! Isolation of protoporphyrin IX from brown eggshells and its detection by optical spectroscopy and chemiluminescence," Journal of Chemical Education 88(6), 788-792 (2011).

51. OECD Series on Principles of Good Laboratory Practice (GLP) and Compliance Monitoring, Organisation for Economic Co-operation and Development, Paris, France, 1998-2019 (accessed 14.02.2020).

52. E. Zherebtsov, V. Dremin, A. Popov, A. Doronin, D. Kurakina, M. Kirillin, I. Meglinski, and A. Bykov, "Hyperspectral imaging of human skin aided by artificial neural networks," Biomedical Optics Express 10(7), 3545-3559 (2019). 